\title{
Experimental Measurement of Overhead Associated with Active Probing of Wireless Mesh Networks
}

\author{
Brian Keegan \\ Technological University Dublin, brian.x.keegan@tudublin.ie \\ Karol Kowalik \\ Technological University Dublin, Karol.kowalik@tudublin.ie
}

Follow this and additional works at: https://arrow.tudublin.ie/commcon

Part of the Electrical and Computer Engineering Commons

\section{Recommended Citation}

Keegan, B. \& Kowalik, K. (2007) Experimental measurement of overhead associated with active probing of wireless mesh networks. IEEE International Conference on Signal Processing and Communications (ICSPC 2007), Dubai, November, 2007.

This Conference Paper is brought to you for free and open access by the Communications Network Research Institute at ARROW@TU Dublin. It has been accepted for inclusion in Conference papers by an authorized administrator of ARROW@TU Dublin. For more information, please contact arrow.admin@tudublin.ie, aisling.coyne@tudublin.ie,gerard.connolly@tudublin.ie.

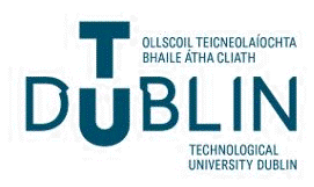




\title{
EXPERIMENTAL MEASUREMENT OF OVERHEAD ASSOCIATED WITH ACTIVE PROBING OF WIRELESS MESH NETWORKS
}

\author{
Brian Keegan, Karol Kowalik and Mark Davis \\ Communications Network Research Institute, \\ Dublin Institute of Technology, Ireland
}

\begin{abstract}
Wireless Mesh Networks (WMNs) represent the next generation wireless networks. The increased capacity of WMNs means that they are now capable of providing backhaul services traditionally maintained by wired networks. The attraction of WMNs is their ease of deployment and ability to self organise, self configure, and self heal. In order to successfully achieve this objective careful consideration must be applied when constructing a WMN. In order to optimise the operation of the network, data should traverse the network by means of the most efficient route. Characterisation and path selection will be determined by the routing algorithm coupled with the link cost metrics. In this paper we experimentally investigate the overhead associated with the estimation of the link quality using the Estimated Transmission Time (ETT) metric.
\end{abstract}

Index Terms - Wireless Mesh, ETT, Active Probing, Overhead

\section{INTRODUCTION}

The minimal configuration of wireless mesh networks (WMNs) makes them a very attractive alternative to current wireless infrastructures. In order to fully exploit the advantages of deploying such a flexible network, one which is capable of self organising, self configuring and self healing, the network must be capable of characterising and optimising links in order to discover efficient routes. In order to achieve this goal the selection of the routing algorithm and performance metric are crucial. Furthermore, the method of characterising the network performance must also be carefully considered. The choice of technique employed by the network designer may impact on the utilisation of the network itself.

Routing protocols can be divided into two categories; active and reactive. Active routing protocols generally work by periodically broadcasting and maintaining routing tables. This type of protocol produces a significant amount of overhead and consequently consumes a large fraction of the available bandwidth. Reactive routing protocols attempt to minimise the impact on the network by gathering information only when necessary. However, the characterisation of a network is carried out by obtaining performance metrics by means of active or passive techniques. With active probing there will be an overhead associated with the broadcast of probe requests. Broadcasting probe requests, however, will have both a direct and indirect overhead. In this paper we attempt to determine the associated overhead associated with using the active probing technique Estimated Transmission Time (ETT) [4]. We compare the performance of a wireless mesh network when no-probes are broadcast to using ETT for $802.11 \mathrm{~b}$ and $802.11 \mathrm{~g}$. We review both the direct and indirect overhead associated with broadcasting ETT probes and accessing the medium.

\section{WMN ROUTING}

One of the basic elements of a WMN is that it utilizes a routing protocol which provides redundancy. In order to achieve this, the routing protocol must select a path set out by the designer. In order for nodes to successfully communicate with each other they must gather information regarding the network topology. This is generally achieved either reactively or proactively. Reactive methods have proven to be more successful for WMNs if such networks are highly dynamic and nodes are allowed to roam. Among the most commonly used reactive protocols are AODV [1] and DSR [2] both of which employ a minimum hop count.

Ad hoc On-Demand Distance Vector (AODV) routing protocol allows nodes to obtain routes, only when necessary, by broadcasting query request packets. Its principal concern is to discover a route with the minimum number of hops. AODV attempts to reduce the overhead by minimizing the number of messages. This is achieved by making use of route sequence numbers thus avoiding loops. It also features a mechanism dealing with broken links and minimising the number of requests sent.

Dynamic Source Routing (DSR), like AODV, is a protocol which operates on demand. This method minimises the overhead by reacting only when route discovery is necessary. Route discovery probe packets are used to determine the route from source to destination. Routed packets contain the address of each node it will traverse in order to get to its destination. 


\section{LINK COST}

In order to characterise and to ultimately optimise the link performance it is necessary to gather a number of performance metrics which amongst others may include delay, signal strength, packet loss and throughput. These metrics may be gathered by employing active probing or passive monitoring methods. Actively probing the network generally requires accessing the wireless network and broadcasting probe requests. Passively monitoring (i.e. switching the wireless card to RFMON mode) on the other hand requires no such access. Instead, by utilising a passive method, wireless nodes are capable of intercepting the transmission activities of neighbouring radios and hence carry out non-intrusive measurements. Once the performance metrics have been processed a link cost can be obtained, hence allowing the link cost function to optimise the utilisation of the wireless medium, adapt to changes in wireless environment, minimise contention between data flows and select stable high throughput paths with low associated delays.

\section{OPERATION OF ETT METRIC}

The Estimated Transmission Time (ETT) is a link cost performance metric derived from Expected Transmission Count (ETX) [3]. The ETX of a link is determined by the forward, $p_{f}$ and reverse, $p_{r}$ delivery ratios. The expected number of transmissions is given as:

$$
E T X=\frac{1}{p_{f} \times p_{r}}
$$

The ETT extends on ETX by not only predicting the amount of time required for a packet to successfully traverse a route, but also observing the highest usable bit rate of each link and the probability of successful delivery at that rate. A simplified description of the ETT which reflects time spent analysing the channel and does not include back-off time is described in [5]. If we let $S$ and $B$ denote the packet size and the link data rate respectively,then;

$$
E T T=E T X \times \frac{S}{B}
$$

ETT uses periodic broadcast packets of two sizes. Small packet sizes of 60 bytes are always transmitted at $1 \mathrm{Mbps}$ and correspond to ACKs. Large packet sizes of 1500 bytes are broadcast at various rates and correspond to data. This means that when using $802.11 \mathrm{~b}$ large packets will be broadcast at 4 different rates $(1,2,5.5$ and $11 \mathrm{Mbps}$ ) whereas using $802.11 \mathrm{~g}$ will mean broadcasting at an additional 8 rates $(6,9,12,18,24,36,48$ and $54 \mathrm{Mbps})$. Statistics gathered at each node are based on these broadcasts. Nodes then share this information with neighbouring nodes. The routing protocol determines that the best route is the one with the lowest ETT.
In order to determine the ETT a number of metrics must be obtained. These include the highest-throughput bitrate and delivery probability. The highest-throughput bitrate for a link is deemed to be the product of the delivery probability and the bit rate. The delivery probability is the product of the number of large (1500 byte) packets successfully delivered and the number of small ( 60 byte) packets successfully received in the reverse direction. The ETT for a given link is the expected time to successfully transmit a large 1500 byte packet (including the time for retransmissions based on the delivery probability) at the highest-throughput bit-rate. The route ETT is simply the sum of the ETTs for each link along that route.

As a result, ETT is required to periodically broadcast probe packets to predict link characteristics. While periodically probing the network reduces overhead compared to similar methods [5] by reducing the number of messages sent, the method still incurs a direct and indirect overhead. By sending probe request, ETT consumes a portion of the available bandwidth. Similarly, by accessing the network other nodes which may need to transmit data will have to defer their access until a probing node has finished transmitting. Ultimately, the combined effect of the direct and indirect overhead is to reduce the capacity of the network.

\section{TEST-BED}

The experimental test-bed consists of 16 desktop PCs configured as static wireless nodes. Each node is a DELL Optiplex equipped with a Netgear WAG511 WLAN card. All nodes are running Linux (kernel version 2.6.11) and MadWiFi drivers. The implementation of DSR routing protocol comes from the Roofnet project and it is implemented using the Click Modular Router Software [6]. The Roofnet implementation of ETT requires nodes to broadcast one probe packet at each rate every 3 seconds. Traffic generation is achieved using IPERF [7] to generate and measure UDP traffic between node pairs. Nodes were set up in pairs to send and receive data. The line rate was kept fixed at $11 \mathrm{Mbps}$, in order to eliminate the use of adaptive rate algorithms, so that a comparison could be made between $802.11 \mathrm{~b}$ and $802.11 \mathrm{~g}$ ETT probe packets. Data for each experiment was taken over a 26 hour period and a mean average was obtained.

\section{RESULTS}

In this section we observe the effects of direct and indirect overhead associated with ETT metric. ETT estimates the time required for successful transmission of a packet (including retransmissions) based on measuring delivery rate and throughput. A result of this is that ETT is required to spend a fixed amount of time probing the networking, therefore causing other nodes to defer their access. As well as this ETT probe packets will consume 
available bandwidth in the actual transmission of probe packets. We present results from comparing the throughput when no probe packets are present to when the network is actively probed using ETT with $802.11 \mathrm{~b}$ and $802.11 \mathrm{~g}$ transmission rates. Similarly we present data for the associated direct overhead.

\subsection{Indirect overhead}

In this experiment 16 nodes ( 8 node pairs) transmit and receive data with identical characteristics. Each node transmits UDP traffic at a certain packet size and rate for 600 seconds. After a test is completed, nodes cease transmitting for a set period in order to allow buffers to clear. The packet rate is then incremented and the test is repeated at the new rate. This process is repeated up to a maximum rate. Once all tests have been run for a given packet size (normally 15 packet rates) the series of tests are repeated a total of 10 times. This amounts to an experiment lasting approximately 26 hours. Repeating the tests in this manner allows us to determine the mean average values for throughput, packet loss and jitter.

\subsubsection{Throughput for 1470 byte packets}

In Figure 1 we observe the throughput when we send large packets of 1470 bytes. We can see from the curves that for all three scenarios (no-probes, 802.11b \& $802.11 \mathrm{~g}$ ) the throughput increases linearly as expected up to saturation. There is, however, a minor difference after this point. We can see that when no-probes are used the network throughput gains a slight advantage over $802.11 \mathrm{~b}$ probe packets and a further increase over $802.11 \mathrm{~g}$ probe packets. The further advantage over $802.11 \mathrm{~g}$ probe packets is attributed to the fact that when using ETT over $802.11 \mathrm{~g}$ rates, there is an increase in periodic probes due to the higher transmission rates. This in turn leads to more broadcast messages utilising more of the available bandwidth.

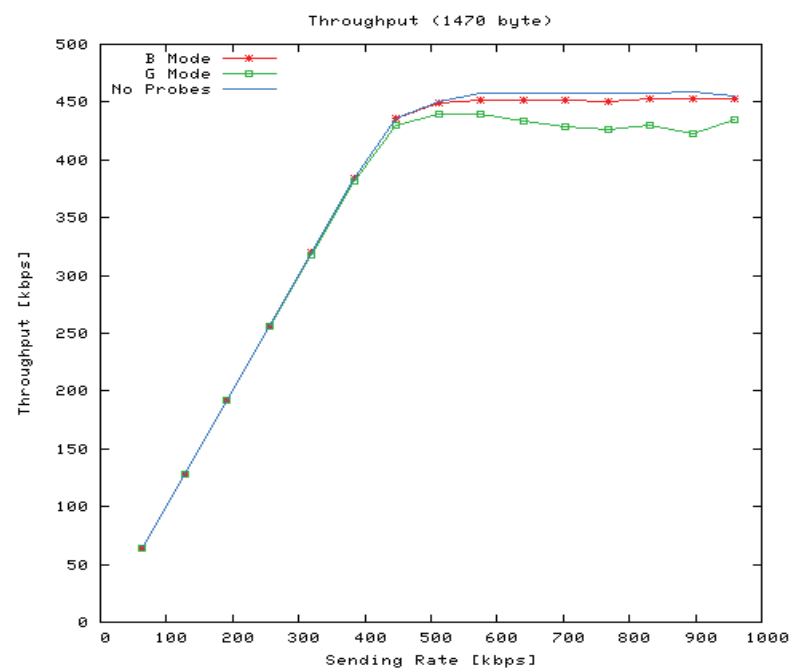

Figure 1. Indirect overhead 1470 byte packet

\subsubsection{Throughput for 64 byte packets}

Our next set of results presents the performance when using a smaller packet size of 64 bytes. In Figure 2 we can see that the effects of the ETT probe packets become more apparent. Again, the throughput increases at a similar rate until saturation. We can see, however, at this point there is a more noticeable difference between the maximum throughputs. A smaller packet size means an increase in the number of packets sent, as a result the number of ETT probe packets will increase. We can see from the figure that using ETT probe requests over $802.11 \mathrm{~g}$ rates will heavily affect the throughput performance when using a smaller packet size.

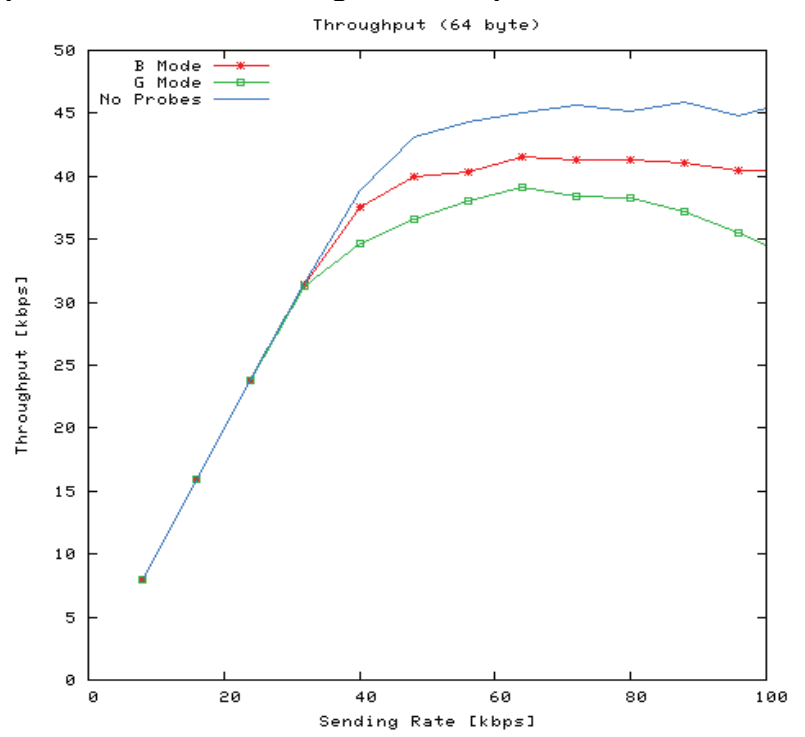

Figure 2. Indirect overhead 60 byte packet

Table 1 outlines the percentage gains over $802.11 \mathrm{~b}$ and $802.11 \mathrm{~g}$ for both large (1470 byte) and small (64 byte) packet sizes.

Table 1. No-Probes percentage gain

\begin{tabular}{|c|c|c|}
\hline & $802.11 \mathrm{~b}$ Probes & $802.11 \mathrm{~g}$ Probes \\
\hline 1470 byte & $2 \%$ & $7.5 \%$ \\
\hline 64 byte & $10 \%$ & $26 \%$ \\
\hline
\end{tabular}

\subsection{Direct overhead}

Section 6.1 illustrated the effects of ETT probe packets on the network. This indirect overhead is a result of ETT probe packets utilising the available bandwidth at different rates. However, the direct overhead associated with accessing the medium is not immediately obvious. When an ETT probe packet gains access to the medium, neighbouring nodes will have to defer their access for a certain amount of time due to fixed time intervals required to transmit probe packets. Each ETT probe request consumes a time interval $t_{i}$;

$$
t_{i}=\left(\frac{\text { frame size }}{\text { transmission rate }}\right)+D I F S
$$


The percentage of time consumed, $T_{c}$ over a period $T$ to actively probe the medium is then given by;

$$
T_{c}=\frac{\sum_{i} t_{i}}{T} \times 100 \%
$$

Figure 3 illustrates the percentage of time a node spends accessing the medium using probe packets for $802.11 \mathrm{~b}$ and $802.11 \mathrm{~g}$. The effect of using ETT is that active probe request must gain access to the medium. Both $802.11 \mathrm{~b}$ and $802.11 \mathrm{~g}$ transmit probe requests at $1 \mathrm{Mbps}$ (which correspond to ACKs) as well as at each of the available bit rates. While $802.11 \mathrm{~b}$ probe requests occur at 4 different bit rates $802.11 \mathrm{~g}$ probe requests occur at 12 different bit rates. It can be clearly seen in the figure how the percentage of time spent issuing probe requests for $802.11 \mathrm{~g}$ bit rates is greater than that of $802.11 \mathrm{~b}$. This time spent accessing the medium for probe requests may have been utilised by neighbouring nodes requiring to forward data along a route.

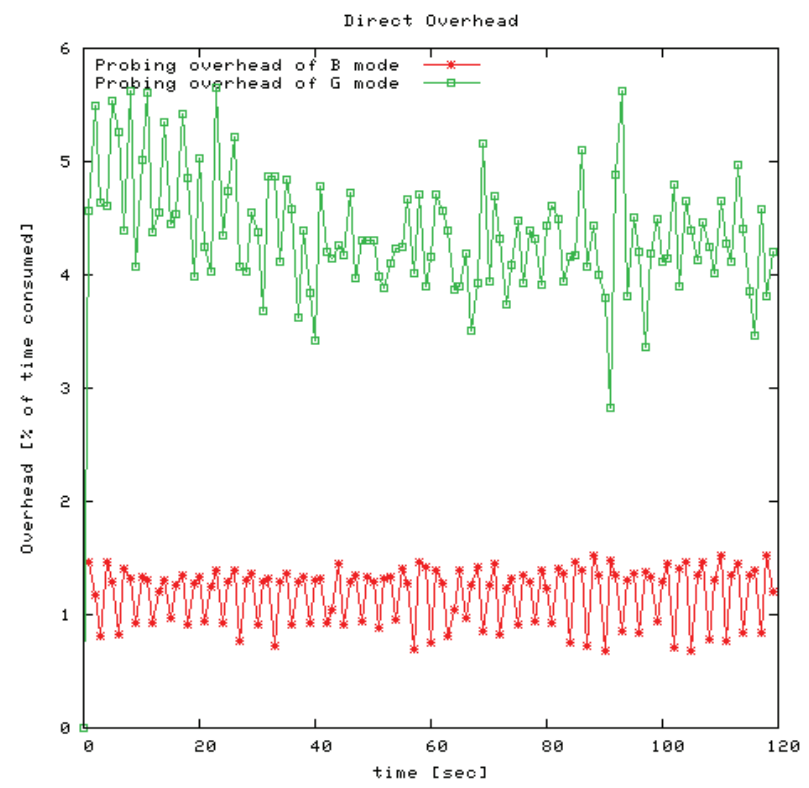

Figure 3. Direct overhead

\section{CONCLUSIONS}

In this paper we have analysed the overhead (both direct and indirect) associated with using active probing for the Estimated Transmission Time (ETT) metric. The experimental analysis compared the throughput for sending large (1470 byte) and small (64 byte) packets at a fixed rate of $11 \mathrm{Mbps}$ for three different scenarios; noprobes, probing at $802.11 \mathrm{~b}$ rates and probing at $802.11 \mathrm{~g}$ rates. We then observed the effects of direct overhead by showing the percentage time spent by a node accessing the medium in order to broadcast probe packets. The findings show how the disadvantage of using active probing become more apparent at lower packet rates as well as being penalised when probing at the higher rates associated with $802.11 \mathrm{~g}$.
The Estimated Transmission Time metric actively probes the medium in order to characterise the link performance. In order to attempt to reduce the overhead ETT minimises the amount of messages sent. However, the very nature of actively probing the network means that there will be an unavoidable minimum amount of associated overhead. As ETT probes at all available bit rates (in order to characterise the link quality at each of these rates) the resulting overhead generated by using the increased number of bit rates available by $802.11 \mathrm{~g}$ becomes quite significant. Therefore, a more efficient method of gaining link characteristics would be a passive monitoring technique as outlined in [8] and implemented in [9]. A passive technique is independent of load and incurs neither a direct nor an indirect overhead.

\section{ACKNOWLEDGEMENTS}

The authors gratefully acknowledge the support of Enterprise Ireland (under the Commercialisation Fund 2006) and Science Foundation Ireland (under the grant 03/IN3/I396.

\section{REFERENCES}

[1] C. Perkins, E. Belding-Royer, and S. Das. RFC 3561: Ad hoc On-Demand Distance Vector (AODV) Routing, July 2003.

[2] David B. Johnson, David A. Maltz, and Yih-Chun $\mathrm{Hu}$. The Dynamic Source Routing protocol for mobile ad hoc networks (DSR), RFC 4728, July 2004.

[3] Douglas S. J. De Couto, Daniel Aguayo, John Bicket, and Robert Morris. A High-Throughput Path Metric for Multi-Hop Wireless Routing. Proceedings of the $9^{\text {th }} A C M$ International Conference on Mobile Computing and Networking (MobiCom '03), September 2003.

[4] J. Bicket, D. Aguayo, S. Biswas, and R. Morris. Architecture and Evaluation of an Unplanned 802.11b Mesh Network. MobiCom, August 2005.

[5] R. Draves, J. Padhye, and B. Zill. Routing in Multiradio, Multi-hop Wireless Mesh Networks. ACM MobiCom, September 2004.

[6] Eddie Kohler, Robert Morris, Benjie Chen, John Jannotti, and M. Frans Kaashoek. The Click modular router. ACM Transactions on Computer Systems, 4(18), November 2000.

[7] NLANR/DAST, Iperf Version 1.7.0, Available from http://dast.nlanr.net/Projects/Iperf/\#download

[8] Mark Davis. A Wireless Traffic Probe for Radio Resource Management and QoS Provisioning in IEEE 802.11 WLANs. ACM Symposium on Modeling, Analysis and Simulation of Wireless and Mobile Systems (MSWiM'04), October 2004.

[9] Karol Kowalik, Brian Keegan and Mark Davis, RARE - Resource Aware Routing for mEsh. ICC 2007 